\title{
Existence and stability of solutions of general semilinear elliptic equations with measure data
}

\author{
Laurent Véron \\ Laboratoire de Mathématiques et Physique Théorique \\ Université François-Rabelais, Tours, FRANCE
}

\begin{abstract}
We study existence and stability for solutions of $-L u+g(x, u)=\omega$ where $L$ is a second order elliptic operator, $g$ a Caratheodory function and $\omega$ a measure in $\bar{\Omega}$. We present a unified theory of the Dirichlet problem and the Poisson equation. We prove the stability of the problem with respect to weak convergence of the data.
\end{abstract}

2010 Mathematics Subject Classification. 35J61, 35J66, 28A20.

Key words: Elliptic operators, Borel measures, Marcinkiewicz spaces, $\Delta_{2}$ condition.

\section{Introduction}

Let $\Omega$ be a smooth bounded domain of $\mathbb{R}^{N}, L$ a uniformly elliptic second order differential operator in divergence form with Lipschitz continuous coefficients and $g$ is a real valued Caratheodory function defined in $\Omega \times \mathbb{R}$. If $\omega$ is a Radon measure on $\bar{\Omega}$, we study existence and stability of solutions of the generalized equation

$$
-L u+g(x, u)=\omega
$$

in $\bar{\Omega}$. Precise assumptions are made on the coefficients of $L$ so that uniqueness holds. A fundamental contribution is made by Benilan and Brezis [6], 3] who study the case where $L=\Delta$ and $g: \mathbb{R} \mapsto \mathbb{R}$ is nondecreasing and positive on $\mathbb{R}_{+}$: if $\mu$ is a bounded measure in $\Omega$ and $g$ satisfies the subcriticality assumption

$$
\int_{1}^{\infty}(g(s)-g(-s)) s^{-2 \frac{N-1}{N-2}} d s<\infty
$$

then there exists a unique function $u \in L^{1}(\Omega)$ such that $g \circ u \in L^{1}(\Omega)$ (where $g \circ u(x)=g(x, u(x))$ ) satisfying

$$
\int_{\Omega}(-u \Delta \zeta+g \circ u \zeta) d x=\int_{\Omega} \zeta d \mu
$$

for any $\zeta \in C_{0}^{2}(\Omega)$.

The boundary value problem with measures is first investigated by Gmira and Véron [7]. By adapting the method introduced by Benilan and Brezis they obtain the existence and uniqueness of a weak solution of

$$
\begin{aligned}
-\Delta u+g(u) & =0 & & \text { in } \Omega \\
u & =\lambda & & \text { in } \partial \Omega
\end{aligned}
$$

when $\lambda$ is a Radon measure. They assume that $g$, always nondecreasing, satisfies the boundary subcriticality assumption

$$
\int_{1}^{\infty}(g(s)-g(-s)) s^{-\frac{2 N}{N-2}} d s<\infty
$$


and prove the existence and uniqueness of a weak solution to (1.4). For this problem, in the integral identity (1.3) the right hand-side is replaced by $-\int_{\partial \Omega} \zeta_{\mathbf{n}} d \lambda$ (where $\zeta_{\mathbf{n}}=\nabla u . \mathbf{n}$ is the outward normal derivative on $\partial \Omega$ ).

In 13. Véron extends Benilan-Brezis results in replacing $\Delta$ by a general uniformly elliptic second order differential operator with smooth coefficients. If $g$ is nondecreasing and satisfies, for some $\alpha \in[0,1]$, the $\alpha$-subcriticality assumption,

$$
\int_{1}^{\infty}(g(s)-g(-s)) s^{-2 \frac{N+\alpha-1}{N+\alpha-2}} d s<\infty
$$

then if $\mu$ belongs to $\mathfrak{M}_{\rho^{\alpha}}(\Omega)$, which means

$$
\|\mu\|_{\mathfrak{M}_{\rho^{\alpha}}}:=\int_{\bar{\Omega}} \rho^{\alpha} d|\mu|<\infty
$$

where $\rho(x):=\operatorname{dist}(x, \partial \Omega)$, there exists a unique $u \in L^{1}(\Omega)$ such that $g(u) \in L_{\rho}^{1}(\Omega)$ satisfying

$$
\int_{\Omega}\left(-u L^{*} \zeta+g(u) \zeta\right) d x=\int_{\Omega} \zeta d \mu \quad \forall \zeta \in C_{c}^{1, L^{*}}(\bar{\Omega}) .
$$

where

$$
C_{c}^{1, L^{*}}(\bar{\Omega})=\left\{\zeta \in C^{1}(\bar{\Omega}): \zeta=0 \text { on } \partial \Omega, L^{*} \zeta \in L^{\infty}(\Omega)\right\},
$$

where $L^{*}$ is the adjoint operator to $L$. Furthermore he proves the weak stability of the problem. it means that if $u_{n}$ is a set of solutions of

$$
\begin{aligned}
-L u_{n}+g\left(u_{n}\right) & =\mu_{n} & & \text { in } \Omega \\
u_{n} & =0 & & \text { in } \partial \Omega
\end{aligned}
$$

for a sequence of measure $\left\{\mu_{n}\right\}$ such that

$$
\lim _{n \rightarrow \infty} \int_{\Omega} \zeta d \mu_{n}=\int_{\Omega} \zeta d \mu
$$

for all $\zeta \in C(\bar{\Omega})$ verifying $\sup _{\Omega} \rho^{-\alpha}|\zeta|<\infty$, then $u_{n} \rightarrow u$ where $u$ satisfies (1.1). However, a careful observation of the existence and stability statements proved in [13, Th 3.7, Cor 3.8] shows that the result is slightly stronger than the one stated since it implies the following:

Let $\alpha \in[0,1]$ and $g: \mathbb{R} \mapsto \mathbb{R}$ be continuous function which satisfies the $\alpha$-subcriticality assumption (1.6). If $\left\{\mu_{n}\right\}$ is a sequence of Radon measures in $\bar{\Omega}$ such that

$$
\int_{\bar{\Omega}} \rho^{\alpha} d\left|\mu_{n}\right| \leq M
$$

for some $M>0$ and (1.11) holds for $\zeta$ such that $\rho^{-\alpha} \zeta \in C(\bar{\Omega})$, then the corresponding solution $u_{n}$ of (1.10) converges to the solution $u$ of (1.1). In particular, if $\alpha=1$, it contains the case where there exists a Radon measure $\lambda$ on $\partial \Omega$ such that

$$
\lim _{n \rightarrow \infty} \int_{\Omega} \zeta d \mu_{n}=-\int_{\partial \Omega} \zeta_{\mathbf{n}} d \lambda \quad \forall \zeta \in C_{c}^{1}(\bar{\Omega})
$$


The case where the nonlinearity $g$ depends on the $\rho(x)$ variable has investigated by Marcus [8]. If $g(x, r) \operatorname{sign} \mathrm{r} \leq \rho(\mathrm{x})^{\beta} \tilde{\mathrm{g}}(|\mathrm{r}|) \operatorname{sign} \mathrm{r}$ for some $\beta>-2$ and $\tilde{g}$ satisfying a subcriticality assumption

$$
\int_{1}^{\infty}(\tilde{g}(s)-\tilde{g}(-s)) s^{-\frac{2 N+\beta-1}{N-1}} d s<\infty
$$

then there exists a weak solution to problem (1.4) for any Radon measure $\lambda$. Furthermore stability holds.

The subcriticality is a key hypothesis in all the previous results: essentially it means that the problem can be solved for any measure if it can be solved for a Dirac measure. The different integral assumptions are just the transcription that the fact that $g$ of the fundamental solution of the associated linear equation is integrable for a suitable measure associated to the distance function $\rho$.

The aim of this article is twofold: 1- to unify the problems for measures in $\Omega$ and on $\partial \Omega$; 2 - to present under the form of an integrability condition a classical sufficient condition of solvability which has the advantage of being a natural extension to the supercritical case the previous subcriticality assumptions and to provide new results results of existence and stability for (1.1) in the spirit of [13]. A function $g: \Omega \times \mathbb{R} \mapsto \mathbb{R}$ belongs to the class $G_{h, \Psi}$ if it is a Caratheodory function and there exist a continuous and nondecreasing function $\tilde{g}: \mathbb{R} \mapsto \mathbb{R}$ vanishing at 0 , a locally integrable nonnegative function $h$ defined in $\Omega$ and a nonnegative continuous nonincreasing function $\Psi:[0, \infty) \mapsto[0, \infty)$, such that

$$
|g(x, r)| \leq h(x)|\tilde{g}(r)| \quad \forall(x, r) \in \Omega \times \mathbb{R},
$$

and the $\Psi$-integrability condition holds, i.e.

$$
-\int_{0}^{\infty}(\tilde{g}(s)-\tilde{g}(-s)) d \Psi(t) d s<\infty .
$$

Let $G$ and $K$ be respectively the Green and Poisson kernels corresponding to the operator $L$ in $\Omega$ and $\mathbb{G}[$.$] and \mathbb{K}[$.$] the corresponding potential operators. The natural subcritical assumptions$ in the framework of Marcus's results (with $h$ instead of $\rho^{\beta}$ ) for solving

$$
\begin{array}{rlrl}
-L u+g(x, u) & =\mu & & \text { in } \Omega \\
u=\lambda & & \text { in } \partial \Omega
\end{array}
$$

would be

$$
\int_{1}^{\infty}(\mathbb{G}[|\mu|]+\mathbb{K}[|\lambda|]) h(x) \rho(x) d x<\infty .
$$

However this type of condition is not satisfactory since it may not hold if $\mu$ and $\lambda$ are merely integrable functions since the problem admits always weak solutions. More generally it does not define a clear class of measures for which we can solve problem (1.17). We introduce new classes of Radon measures whose Green and Poisson potentials belong to a weighted Marcinkiewicz 
space-type space. Let $\Psi$ be a continuous nonincreasing and nonnegative function defined on $[0, \infty)$ and $m$ is a bounded positive Borel measure in $\Omega$ and denote

$$
M_{m}^{\Psi}(\Omega):=\left\{f \in \mathcal{B}(\Omega): \exists C>0 \text { s.t. } \int_{\lambda_{f}(t)} d m(x) \leq C \Psi(t), \forall t>0\right\}
$$

where $\mathcal{B}(\Omega)$ denotes the space of Borel functions in $\Omega$ and $\lambda_{f}(t)=\{x \in \Omega:|f(x)|>t\}$. The main results of this article are the two next statements:

Theorem A Let $g$ be an element of the class $G_{h, \Psi}$ with $\rho h \in L^{1}(\Omega)$. Then for any $\mu \in \mathfrak{M}_{\rho}(\Omega)$ and $\lambda \in \mathfrak{M}(\partial \Omega)$ such that $\mathbb{G}[|\mu|]$ and $\mathbb{K}[|\lambda|]$ belong to $M_{\rho h}^{\Psi}(\Omega)$, there exists a solution to problem (1.17). If $r \mapsto g(x, r)$ is nondecreasing for a.e. $x \in \Omega$, this solution is unique.

Actually we shall introduce a unique formulation for the data $(\mu, \lambda)$ as a unique measure $\omega$ on $\bar{\Omega}$ which allows to replace (1.17) by (1.1), and a unique assumption on the extended Green operator $\overline{\mathbb{G}}[|\omega|]$. We prove in particular the following:

Theorem B Assume the assumptions on $h, \Psi$ and $g$ of Theorem $A$ are satisfied and $r \mapsto g(x, r)$ is nondecreasing. If $\left\{\left(\omega_{n}\right\}\right.$ is a sequence of measures in $\mathfrak{M}_{\rho}(\bar{\Omega})$ which converges to $\omega \in \mathfrak{M}_{\rho}(\bar{\Omega})$ in the sense that

$$
\int_{\bar{\Omega}} \zeta d \omega_{n} \rightarrow \int_{\bar{\Omega}} \zeta d \omega
$$

for any $\zeta$ such that $\rho^{-1} \zeta \in C(\bar{\Omega})$ and if the $\overline{\mathbb{G}}\left[\left|\omega_{n}\right|\right]$ are bounded in $M_{\rho h}^{\Psi}(\Omega)$, then the corresponding solutions $u_{\omega_{n}}$ of problem (1.10) converges to the solution $u_{\omega}$ of problem (1.1). If $g$ satisfies the $\Delta_{2}$ conditions, the convergence remains valid if only the $\overline{\mathbb{G}}\left[\left|\omega_{s n}\right|\right]$ are bounded in $M_{\rho h}^{\Psi}(\Omega)$, where $\omega_{\text {sn }}$ denotes the singular parts of $\omega_{n}$.

\section{Linear equations and measures}

Since $\partial \Omega$ is $C^{2}$, there exists $\delta_{0}>0$ such that, If $x \in \Omega$ is such that $\rho(x) \leq \delta_{0}$, there exists a unique $\sigma:=\sigma(x) \in \partial \Omega$ such that $|x-\rho(x)|=\rho(x)$. For $\delta>0$ we denote

$\Omega_{\delta}:=\{x \in \Omega: \rho(x)>\delta\}, \Omega_{\delta}^{\prime}:=\{x \in \Omega: \rho(x)<\delta\}, \Sigma_{\delta}:=\{x \in \Omega: \rho(x)=\delta\}, \Sigma:=\Sigma_{0}=\partial \Omega$.

The mapping $x \mapsto(\rho(x), \sigma(x))$ is a $C^{1}$ diffeomorphism from $\overline{\Omega_{\delta_{0}}^{\prime}}$ onto $\left[0, \delta_{0}\right] \times \Sigma$.

\subsection{Weighted measures on $\bar{\Omega}$}

We denote by $\mathfrak{M}(\Omega)$ the set of Radon measures in $\Omega$. If $\alpha \in[0,1]$, we denote by $\mathfrak{M}_{\rho^{\alpha}}(\Omega)$ the subset of $\mathfrak{M}(\Omega)$ of measures such that

$$
\|\mu\|_{\mathfrak{M}_{\rho^{\alpha}}}:=\int_{\Omega} \rho^{\alpha} d|\mu|<\infty
$$

We also set

$$
\left.C_{\alpha}(\bar{\Omega}):=\left\{\zeta \in C(\Omega): \rho^{-\alpha} \zeta \in C(\bar{\Omega})\right\}\right\}
$$


with norm

$$
\|\zeta\|_{C_{\alpha}}:=\sup _{x \in \bar{\Omega}} \rho^{-\alpha}(x)|\zeta(x)| .
$$

Thus, if $\mu \in \mathfrak{M}_{\rho^{\alpha}}(\Omega)$ and $\zeta \in C_{\alpha}(\bar{\Omega})$, there holds

$$
\left|\int_{\Omega} \zeta d \mu\right| \leq\|\mu\|_{\mathfrak{M}_{\rho^{\alpha}}}\|\zeta\|_{C_{\alpha}}
$$

Furthermore, since

$$
\int_{\Omega_{\delta_{0}}} \rho^{\alpha} d|\mu|+\sum_{n=1}^{\infty} \int_{\left\{2^{-n} \delta_{0}<\rho \leq 2^{1-n} \delta_{0}\right\}} \rho^{\alpha} d|\mu|=\int_{\Omega} \rho^{\alpha} d|\mu|<\infty
$$

there holds

$$
\lim _{\delta \rightarrow 0} \int_{\Omega_{\delta}^{\prime}} \rho^{\alpha} d|\mu|=0
$$

We say that a sequence $\left\{\mu_{n}\right\} \subset \mathfrak{M}_{\rho^{\alpha}}(\Omega)$ converges weakly to $\mu \in \mathfrak{M}_{\rho^{\alpha}}(\Omega)$ if, for any $\zeta \in C_{\alpha}(\bar{\Omega})$, there holds

$$
\lim _{n \rightarrow \infty} \int_{\Omega} \zeta d \mu_{n}=\int_{\Omega} \zeta d \mu
$$

However, the left-hand side expression of (2.26) may exist but not being a Radon measure in $\Omega$. Therefore we define a more general set of linear functionals on $C_{\alpha}$

Definition 2.1 We denote by $\mathfrak{M}_{\rho^{\alpha}}(\bar{\Omega})$ the set of continuous linear functionals $\omega$ on $C_{\alpha}(\bar{\Omega})$ such that there exists a sequence $\left\{\mu_{n}\right\} \subset \mathfrak{M}_{\rho^{\alpha}}(\Omega)$ which converges weakly to $\omega$.

The natural norm in $\mathfrak{M}_{\rho^{\alpha}}(\bar{\Omega})$ is

$$
\|\omega\|_{\mathfrak{M}_{\rho} \alpha}(\bar{\Omega})=\sup \left\{|\omega(\zeta)|: \zeta \in C_{\alpha}(\bar{\Omega}),\|\zeta\|_{C_{\alpha}} \leq 1\right\}
$$

Proposition 2.2 If $\omega \in \mathfrak{M}_{\rho^{\alpha}}(\bar{\Omega})$, its restriction to $C_{c}(\Omega)$ is a Radon measure, denoted by $\mu$, which belongs to $\mathfrak{M}_{\rho^{\alpha}}(\Omega)$. Furthermore, there exists a Radon measure $\lambda$ on $\partial \Omega$ such that

$$
\omega(\zeta)-\int_{\Omega} \zeta d \mu=\int_{\partial \Omega} \psi\left\lfloor_{\partial \Omega} d \lambda \quad \forall \zeta \in C_{\alpha}(\bar{\Omega}) \text { and } \psi=\rho^{-\alpha} \zeta \in C(\bar{\Omega}) .\right.
$$

Proof. Since $\omega$ is continuous, there exists $C>0$ such that

$$
|\omega(\zeta)| \leq C\|\zeta\|_{C_{\alpha}} \quad \forall \zeta \in C_{\alpha}(\bar{\Omega}) .
$$

This holds in particular if $\zeta \in C_{c}(\Omega)$ and proves that the restriction of $\omega$ to $C_{c}(\Omega)$ is a Radon measure that we denote by $\mu$ (as well as the associated Borel measure in $\Omega$ ) and

$$
\omega(\zeta)=\int_{\Omega} \zeta d \mu \quad \forall \zeta \in C_{c}(\Omega)
$$


Let $\left\{\mu_{n}\right\} \subset \mathfrak{M}_{\rho^{\alpha}}(\Omega)$ such that

$$
\lim _{n \rightarrow \infty} \int_{\Omega} \zeta d \mu_{n}=\omega(\zeta) \quad \forall \zeta \in C_{\alpha}(\bar{\Omega})
$$

By the Banach-Steinhaus theorem there exists $C>0$ such that $\left\|\mu_{n}\right\|_{\mathfrak{M}_{\rho^{\alpha}}} \leq C$ for all $n \in \mathbb{N}$. Since for $\zeta \in C_{c}(\Omega)$,

$$
\omega(\zeta)-\int_{\Omega} \zeta d \mu=\lim _{n \rightarrow \infty} \int_{\Omega} \zeta d\left(\mu_{n}-\mu\right)
$$

and

$$
\left|\int_{\Omega} \zeta d\left(\mu_{n}-\mu\right)\right| \leq 2 C\|\zeta\|_{C_{\alpha}}
$$

it follows that $\left\{\lambda_{n}\right\}:=\left\{\rho^{\alpha}\left(\mu_{n}-\mu\right)\right\}$ is a sequence of Radon measures on $\Omega$, bounded in $\mathfrak{M}_{\rho^{\alpha}}(\Omega)$ and such that

$$
\lim _{n \rightarrow \infty} \int_{\Omega} \zeta d \lambda_{n}=0 \quad \forall \zeta \in C_{c}(\Omega)
$$

Therefore there exists a Radon measure $\lambda$ with support in $\partial \Omega$ and a subsequence $\lambda_{n_{k}}$ such that

$$
\lim _{n \rightarrow \infty} \int_{\Omega} \psi d \lambda_{n_{k}}=\int_{\partial \Omega} \psi\lfloor\partial \Omega d \lambda
$$

which implies (2.28).

Corollary 2.3 The mapping $T: \mathfrak{M}_{\rho^{\alpha}}(\Omega) \times \mathfrak{M}(\partial \Omega) \mapsto \mathfrak{M}_{\rho^{\alpha}}(\bar{\Omega})$ defined by

$$
T[\mu, \lambda](\zeta)=\int_{\Omega} \zeta d \mu+\int_{\partial \Omega} \psi\left\lfloor_{\partial \Omega} d \lambda \quad \forall \zeta \in C_{\alpha}(\bar{\Omega}) \text { and } \psi=\rho^{-\alpha} \zeta \in C(\bar{\Omega}) .\right.
$$

is one to one. Furthermore

$$
\max \left\{\|\mu\|_{\mathfrak{M}_{\rho^{\alpha}}(\Omega)},\|\lambda\|_{\mathfrak{M}_{(\partial \Omega)}}\right\} \leq\|T[\mu, \lambda]\|_{\mathfrak{M}_{\rho^{\alpha}}(\bar{\Omega})} \leq\|\mu\|_{\mathfrak{M}_{\rho^{\alpha}}(\Omega)}+\|\lambda\|_{\mathfrak{M}(\partial \Omega)} .
$$

Proof. The mapping $T$ is onto from Proposition 2.2. The mapping $T$ is one to one since if $T[\mu, \lambda]=0$, then $\mu=0$ and $\int_{\partial \Omega} \psi \iota_{\partial \Omega} d \lambda=0$ for any $\psi \in C(\bar{\Omega})$. This implies $\lambda=0$. The right-hand side inequality (2.31) is clear since sup $\mid \psi\left\lfloor_{\partial \Omega} \mid \leq\|\zeta\|_{C_{\alpha}}\right.$. Because of (2.25)

$$
\int_{\Omega} \rho^{\alpha} d|\mu|=\sup \left\{\int_{\Omega} \zeta d \mu: \zeta \in C_{c}(\Omega),\|\zeta\|_{C_{\alpha}} \leq 1\right\}
$$

This implies

$$
\|\mu\|_{\mathfrak{M}_{\rho^{\alpha}}(\Omega)} \leq\|T[\mu, \lambda]\|_{\mathfrak{M}_{\rho^{\alpha}}(\bar{\Omega})}
$$

If $\phi \in C(\partial \Omega)$ is such that $|\phi| \leq 1$ and $\Phi$ is its harmonic lifting in $\Omega$, the function $\zeta=\rho^{\alpha} \Phi$ belongs to $C_{\alpha}(\bar{\Omega})$ and satisfies $\|\zeta\|_{C^{\alpha}} \leq 1$. Let $\left\{\eta_{n}\right\} \subset C^{\infty}\left(\mathbb{R}^{N}\right)$ such that $0 \leq \eta_{n} \leq 1, \eta_{n}(x)=0$ if $\rho(x) \geq 2 / n, \eta_{n}(x)=1$ if $\rho(x) \leq 1 / n$. Then $\zeta_{n}=\eta_{n} \rho^{\alpha} \Phi$ belongs also to $C_{\alpha}(\bar{\Omega})$ and $\left\|\zeta_{n}\right\|_{C^{\alpha}} \leq 1$. Since

$$
T[\mu, \lambda]\left(\zeta_{n}\right)=\int_{\Omega} \zeta_{n} d \mu+\int_{\partial \Omega} \phi d \lambda
$$


and $\int_{\Omega} \zeta_{n} d \mu \rightarrow 0$ as $n \rightarrow \infty$, we derive

$$
\|T[\mu, \lambda]\|_{\mathfrak{M}_{\rho} \alpha(\bar{\Omega})} \geq \int_{\partial \Omega} \phi d \lambda
$$

This ends to proof.

Remark. If $\lambda$ is a Radon measure on $\partial \Omega$ and we can define its $\delta^{\alpha}$-lifting $\Lambda_{\delta^{\alpha}}[\lambda] \in \mathfrak{M}(\Omega)$ by

$$
\int_{\Omega} \zeta d \lambda_{\delta^{\alpha}}=\delta^{-\alpha} \int_{\Omega} \zeta(\delta, \sigma) d \lambda(\sigma)
$$

Clearly $\lambda_{\delta^{\alpha}} \in \mathfrak{M}_{\rho^{\alpha}}(\Omega)$ and if $\zeta \in C_{\alpha}(\bar{\Omega})$ and $\ell_{\alpha}(\zeta)=-\lim _{\rho \rightarrow 0} \rho^{-\alpha} \zeta$, then $\ell_{\alpha}(\zeta) \in C(\partial \Omega)$, there holds

$$
\lim _{\delta \rightarrow 0} \int_{\Omega} \zeta d \lambda_{\delta^{\alpha}}=\int_{\Sigma} \ell_{\alpha}(\zeta) d \lambda
$$

In the particular case where $\alpha=1 \ell_{\alpha}(\zeta)=\zeta_{\mathbf{n}}:=\lim _{\rho \rightarrow 0} \rho^{-1} \zeta$, and

$$
\lim _{\delta \rightarrow 0} \int_{\Omega} \zeta d \lambda_{\delta}=-\int_{\Sigma} \zeta_{\mathbf{n}} d \lambda
$$

\subsection{The linear operator}

Let $x=\left(x_{1}, \ldots, x_{N}\right)$ the coordinates in $\mathbb{R}^{N}$ and $\Omega$ a bounded domain in $\mathbb{R}^{N}$. We consider the operator $L$ in divergence form defined by

$$
L u:=-\sum_{i, j=1}^{N} \frac{\partial}{\partial x_{i}}\left(a_{i j} \frac{\partial u}{\partial x_{j}}\right)+\sum_{i=1}^{N} b_{i} \frac{\partial u}{\partial x_{i}}-\sum_{i=1}^{N} \frac{\partial}{\partial x_{i}}\left(c_{i} u\right)+d u
$$

where the $a_{i j}, b_{i}$ and $c_{i}$ are Lipschitz continuous and $d$ is bounded and measurable in $\Omega$. We assume that the ellipticity condition

$$
\sum_{i, j=1}^{N} a_{i j}(x) \xi_{i} \xi_{j} \geq a \sum_{i 1}^{N} \xi_{i}^{2} \quad \forall \xi \in \mathbb{R}^{N}
$$

holds for almost $x$ in $\Omega$, for some $a>0$. We also assume the positivity condition

$$
\int_{\Omega}\left(d v+\frac{1}{2} \sum_{i=1}^{N}\left(b_{i}+c_{i}\right) \frac{\partial v}{\partial x_{i}}\right) d x \geq 0 \quad \forall v \in C_{c}^{1}(\Omega), v \geq 0
$$

Under these assumptions, the bilinear form

$$
(u, v) \mapsto A_{L}(u, v)=\int_{\Omega}\left(\sum_{i, j=1}^{N} a_{i j} \frac{\partial u}{\partial x_{j}} \frac{\partial v}{\partial x_{i}}+\sum_{i=1}^{N}\left(b_{i} \frac{\partial u}{\partial x_{i}} v+c_{i} \frac{\partial v}{\partial x_{i}} u\right)+d u v\right) d x
$$


is continuous and coercive on $W^{1,2}(\Omega)$. We define the adjoint operator $L^{*}$ by

$$
L^{*} u:=-\sum_{i, j=1}^{N} \frac{\partial}{\partial x_{j}}\left(a_{i j} \frac{\partial u}{\partial x_{i}}\right)+\sum_{i=1}^{N} c_{i} \frac{\partial u}{\partial x_{i}}-\sum_{i=1}^{N} \frac{\partial}{\partial x_{i}}\left(b_{i} u\right)+d u
$$

We denote by $G=G_{L}$ and $K=K_{L}$ the Green and Poisson kernels corresponding to the operator $L$ in $\Omega$. We recall the following equivalence statement [10], [2]

Proposition 2.4 Assume $\Omega$ has a $C^{2}$ boundary and (2.36) holds. Then there exists a positive constant $C$ such that

$$
C G_{-\Delta} \leq G \leq C^{-1} G_{-\Delta} \quad \text { in } \Omega \times \Omega \backslash D_{\Omega}
$$

where $D_{\Omega}=x \in \Omega \times \Omega: x: \neq y$ and

$$
C K_{-\Delta} \leq K \leq C^{-1} K_{-\Delta} \quad \text { in } \Omega \times \partial \Omega .
$$

\subsection{Linear equation with measure data}

If $m \in \mathfrak{M}_{+}(\Omega)$ is a bounded Borel measure and $\Psi:[0, \infty) \mapsto[0, \infty)$ is continuous and nonincreasing, we define the subset $M_{m}^{\Psi}(\Omega)$ of the set $\mathcal{B}(\Omega)$ of Borel mesurable functions by

$$
M_{m}^{\Psi}(\Omega):=\left\{f \in \mathcal{B}(\Omega): \exists C>0 \text { s.t. } \int_{\lambda_{f}(t)} d m(x) \leq C \Psi(t), \forall t>0\right\}
$$

where

$$
\lambda_{f}(t)=\{x \in \Omega:|f(x)|>t\} .
$$

Notice that $\Psi(t) \leq m(\Omega)$ for $t \geq 0$. Denote

$$
\bar{\lambda}_{f}(t)=\{x \in \Omega:|f(x)| \geq t\} .
$$

Since $\Psi$ is continuous, (2.41) implies

$$
\int_{\bar{\lambda}_{f}(t)} d m(x) \leq C \Psi(t), \forall t>0
$$

If we modify $\Psi$ in order to impose $\Psi(0)=m(\Omega)$, (2.41) is equivalent to

$$
M_{m}^{\Psi}(\Omega):=\left\{f \in \mathcal{B}(\Omega): \exists C>0 \text { s.t. } \int_{\bar{\lambda}_{f}(t)} d m(x) \leq C \Psi(t), \forall t \geq 0\right\}
$$

We denote by $C_{m}^{\Psi}(f)$ the smallest constant $C$ such that (2.41) holds. If $t \mapsto \Psi(t) / \Psi(2 t)$ remains bounded on $[0, \infty), M_{m}^{\Psi}(\Omega)$ is a vector space $f \mapsto C_{m}^{\Psi}(f)$ is a quasi-norm on the quotient space $M_{m}^{\Psi}(\Omega) / \mathcal{R}$ where $\mathcal{R}$ is the equivalence relation $f_{1} \mathcal{R} f_{2} \Longleftrightarrow f_{1}-f_{2}=0 m$-a.e. in $\Omega$. In general $M_{m}^{\Psi}(\Omega)$ is not a vector space

When $\Psi(t)=t^{-p}$ with $p \geq 1$ and $m(x)=\rho(x)^{\alpha}$, with $\alpha \in[0,1]$, we denote by $M_{\rho^{\alpha}}^{p}(\Omega)$ the corresponding Marcinkiewicz space. The following results proved in [5] with $L=-\Delta$ are valid for a general operator $L$ 
Proposition 2.5 Let $\alpha \in[0,1], N \geq 2$. If $\mu \in \mathfrak{M}_{\rho^{\alpha}}(\bar{\Omega})$ and $N+\alpha-2>0$,

$$
\begin{gathered}
\|\mathbb{G}[\mu]\|_{M_{\rho^{\alpha}}^{(N+\alpha) /(N+\alpha-2)}} \leq C\|\mu\|_{\mathfrak{M}_{\rho^{\alpha}}}, \\
\|\nabla \mathbb{G}[\mu]\|_{M_{\rho^{\alpha}}^{(N+\alpha) /(N+\alpha-1)}} \leq C\|\mu\|_{\mathfrak{M}_{\rho^{\alpha}}} .
\end{gathered}
$$

Furthermore, for any $\gamma \in[0,1]$ and $\lambda \in \mathfrak{M}(\partial \Omega)$,

$$
\|\mathbb{K}[\lambda]\|_{M_{\rho \gamma}^{(N+\gamma) /(N-1)}} \leq C\|\lambda\|_{\mathfrak{M}} .
$$

We recall the following result proved in [13, Th 2.9]

Theorem 2.6 Let $\alpha \in[0,1]$. For every $\mu \in \mathfrak{M}_{\rho^{\alpha}}(\Omega)$ and $\lambda \in \mathfrak{M}(\partial \Omega)$, there exists a unique $u:=u_{\mu, \lambda} \in L^{1}(\Omega)$ satisfying

$$
\begin{aligned}
-L u=\mu & \text { in } \Omega \\
u=\lambda & \text { in } \partial \Omega,
\end{aligned}
$$

in the following weak sense

$$
-\int_{\Omega} u L^{*} \zeta d x=\int_{\Omega} \zeta d \mu-\int_{\partial \Omega} \zeta_{\mathbf{n}} d \lambda \quad \forall \zeta \in C_{c^{1, L}}(\bar{\Omega}) .
$$

Furthermore, if $\left\{\left(\mu_{n, \lambda_{n}}\right)\right\}$ is bounded in $\mathfrak{M}_{\rho^{\alpha}}(\Omega) \times \mathfrak{M}(\partial \Omega)$ and converges weakly with respect to $C_{\alpha}(\bar{\Omega}) \times C(\partial \Omega)$ to $(\mu, \lambda) \in \mathfrak{M}_{\rho^{\alpha}}(\Omega) \times \mathfrak{M}(\partial \Omega)$, then $u_{\mu_{n}, \lambda_{n}}$ converges to $u_{\mu, \lambda}$.

Remark. If we define the measure $\omega \in \mathfrak{M}_{\rho^{\alpha}}(\bar{\Omega})$ by $\omega=T[\mu, \lambda]$ (see (2.30) $)$, then it can also be expressed by

$$
\int_{\bar{\Omega}} \zeta d \omega:=\int_{\Omega} \zeta d \mu-\int_{\partial \Omega} \zeta_{\mathbf{n}} d \lambda \quad \forall \zeta \in C_{1}(\bar{\Omega})
$$

since $\zeta \in C_{1}(\bar{\Omega})$ implies that $\zeta_{\mathbf{n}}$ exists on $\partial \Omega$ and is continuous. We define the global Green operator on $\bar{\Omega}$ by

$$
\overline{\mathbb{G}}[\omega]:=\mathbb{G}[\mu])+\mathbb{P}_{L}[\lambda] .
$$

and (2.48) is replaced by the unique equation

$$
-L u=\omega \quad \text { in } \bar{\Omega} .
$$

Then (2.45)-(2.47) with $\alpha=1$ are equivalent to

$$
\|\overline{\mathbb{G}}[\omega]\|_{M_{\rho}^{(N+1) /(N-1)}} \leq C\|\omega\|_{\mathfrak{M}_{\rho}}
$$

Furthermore, we say that $u \in L^{1}(\Omega)$ is a subsolution of (2.52) in $\bar{\Omega}$, if

$$
-\int_{\Omega} u L^{*} \zeta d x \leq \int_{\bar{\Omega}} \zeta d \omega:=\int_{\Omega} \zeta d \mu-\int_{\partial \Omega} \zeta_{\mathbf{n}} d \lambda \quad \forall \zeta \in C_{c}^{1, L^{*}}(\bar{\Omega}), \zeta \geq 0 .
$$

Comparison principle applies, thus $u \leq \overline{\mathbb{G}}[\omega]$. A supersolution is defined similarly.

Remark. If $\omega=T[\mu, \lambda] \in \mathfrak{M}_{\alpha}^{+}(\bar{\Omega})$ its Lebesgue decomposition is $\omega_{r}+\omega_{s}=T\left[\mu_{r}, \lambda_{r}\right]+T\left[\mu_{s}, \lambda_{s}\right]$ where $\mu_{r}$ and $\lambda_{r}$ are the absolutely continuous part with respect to the Hausdorff measures $d \mathcal{H}^{N}$ and $d \mathcal{H}^{N-1}$ and $\mu_{s}$ and $\lambda_{s}$ the respective singular parts. Similarly if $\omega=T[\mu, \lambda]$, then $\omega=\omega^{+}-\omega^{-}$where $\omega^{+}=T\left[\mu^{+}, \lambda^{+}\right]$and $\omega^{-}=T\left[\mu^{-}, \lambda^{-}\right]$. 


\subsection{Regularity results}

We define the class of measures $B_{h}^{p}(\bar{\Omega})$ by

$$
\left.B_{h}^{\Psi}(\bar{\Omega}):=\left\{\omega \in \mathfrak{M}_{\rho}(\bar{\Omega}): \overline{\mathbb{G}}[|\omega|] \in M_{\rho h}^{\Psi} \Omega\right)\right\} .
$$

By Proposition 2.4, this class remains unchanged if we replace $-\Delta$ by $L$ and the Green operator for $L$ by the one of $-\Delta$. If $\Psi(t)=t^{-p}$ and $h=1$, the corresponding class of measures is larger that the usual

$$
\tilde{B}^{p}(\bar{\Omega}):=\left\{\omega \in \mathfrak{M}_{\rho}(\bar{\Omega}): \overline{\mathbb{G}}[|\omega|] \in L_{\rho}^{p}(\Omega)\right\}
$$

which corresponds to negative Besov spaces: if $\omega=T[\mu, \lambda]$, then the regularity results for harmonic functions [9] and solution of Laplace equation [1] yields to

$$
\tilde{B}^{p}(\bar{\Omega})=B^{-\frac{2}{p}, p}(\Omega)
$$

Example 1 If $h(x)=(\rho(x))^{\beta}$, with $\beta>-2$. Then $\omega=T[0, \lambda] \in B_{\rho^{\beta}}^{p}(\bar{\Omega})$ if and only if $\overline{\mathbb{G}}[|\omega|] \in$ $M_{\rho^{\beta+1}}(\Omega)$. This means that $\lambda \in B_{\infty}^{-s, p}(\partial \Omega)$ with $s=(\beta+2) / p$ (see [1] for the definition of $B_{q}^{\alpha, p}$.

\section{The main results}

Definition 3.1 We say that a Caratheodory function $g: \Omega \times \mathbb{R}$ belongs to the class $G_{h, \Psi}$ if there exist a nonnegative function $h \in L_{\rho}^{1}(\Omega)$, a continuous nondecreasing function $\tilde{g}$ defined on $\mathbb{R}_{+}$and vanishing at $r=0$ such that $0 \leq g(x, r) \operatorname{sign} \mathrm{r} \leq \mathrm{h}(\mathrm{x}) \tilde{\mathrm{g}}(|\mathrm{r}|)$ in $\Omega \times \mathbb{R}$ and a continuous nonincreasing function $\Psi:[0, \infty) \mapsto[0, \infty)$ with the property that

$$
-\int_{1}^{\infty} \tilde{g}(s) d \Psi(s)<\infty .
$$

Lemma 3.2 Let $\mu$ be a nonnegative measure in $\mathfrak{M}(\Omega)$ and $g: \Omega \times \mathbb{R} \mapsto \mathbb{R}$ a Caratheodory function such that $0 \leq g(x, r) \operatorname{sign} \mathrm{r} \leq \mathrm{h}(\mathrm{x}) \tilde{\mathrm{g}}(|\mathrm{r}|)$ where $h \in L_{\rho}^{1}(\Omega)$ and $\tilde{g}$ is a continuous and nondecreasing function $\tilde{g}$ defined on $\mathbb{R}_{+}$and vanishing at $r=0$. Then

(i) If $g \in G_{h, \Psi}$ and $\mu \in B_{h}^{\Psi}(\bar{\Omega})$, then $\tilde{g} \circ \overline{\mathbb{G}}[\mu] \in L_{\rho h}^{1}(\Omega)$.

(ii) if $\tilde{g} \circ \overline{\mathbb{G}}[\mu] \in L_{\rho h}^{1}(\Omega)$ and, then $\mu \in B_{h}^{\Psi}(\bar{\Omega})$ and $g \in G_{h, \Psi}$ with $\Psi(s)=\theta_{\lambda_{\bar{G}[\mu]}}(s)$, where $\lambda_{\overline{\mathbb{G}}[\mu]}(s)$ is defined by (2.42) with $f$ replaced by $\overline{\mathbb{G}}[\mu]$ and $\theta_{\lambda_{\bar{G}[\mu]}}(s)=\int_{\lambda_{\overline{\mathbb{G}}[\mu]}(s)} d(\rho h)$.

Proof. This due to the fact that

$$
\int_{\Omega} \tilde{g}(\overline{\mathbb{G}}[\mu]) \rho h d x=-\int_{0}^{\infty} \tilde{g}(s) d \theta_{\lambda_{\overline{\mathbb{G}}[\mu]}}(s) .
$$

Therefore, if $\theta_{\lambda_{\bar{G}[\mu]}}(s) \leq \Psi(s)$, it proves (i). Conversely, if $\Psi(s)=\theta_{\lambda_{\bar{G}[\mu]}}(s)$, then $\mu \in B_{h}^{\Psi}(\bar{\Omega})$ and $g \in G_{h, \Psi}$.

The following existence result extends to one in [13] 
Theorem 3.3 Assume $g$ belongs to the class $G_{h, \Psi}$. Then for any $\omega \in B_{h}^{\Psi}(\bar{\Omega})$ there exists a function $u \in L^{1}(\Omega)$ such that $g \circ u \in L^{1}(\Omega)$ satisfying

$$
\int_{\Omega}\left(-u L^{*} \zeta+g \circ u \zeta\right) d x=\int_{\bar{\Omega}} \zeta d \omega \quad \forall \zeta \in C_{c}^{1, L^{*}}(\bar{\Omega})
$$

Furthermore $u$ is unique if $r \mapsto g(x, r)$ is nondecreasing for a.e. $x \in \Omega$.

Proof. It is essentially [13, Theorem 3.7]. Since $0 \leq g(x, r) \operatorname{sign} \mathrm{r} \leq \mathrm{h}(\mathrm{x}) \tilde{\mathrm{g}}(|\mathrm{r}|)$, we define the following truncation $g_{k}(., r)$ for any $k>0$.

$$
g_{k}(x, r)=g(x, r) \chi_{\Theta_{k}}
$$

where $\Theta_{k}=\{x \in \Omega: h(x) \leq k\}$. Then $0 \leq g(x, r) \operatorname{sign} \mathrm{r} \leq \mathrm{k} \tilde{\mathrm{g}}(|\mathrm{r}|)$ and there exists a solution $u_{k}$ to

$$
-L u_{k}+g_{k} \circ u_{k}=\omega \quad \text { in } \bar{\Omega} .
$$

Actually, in [13, Theorem 3.7] the proof is done with $\mu \in \mathfrak{M}_{\rho^{\alpha}}(\Omega)$ for any $\alpha \in[0,1]$, but due to our definition of measures in $\mathfrak{M}_{\rho^{\alpha}}(\bar{\Omega})$, it is also valid in this case.

Step 2: Convergence when $k \rightarrow \infty$. By Brezis'estimates (see e.g. [13, Th 2.4]), for any $\zeta \in$ $C_{c}^{1, L}(\bar{\Omega}), \zeta \geq 0$, one has

$$
\int_{\Omega}\left(-\left|u_{k}\right| L^{*} \zeta+\operatorname{sign}\left(\mathrm{u}_{\mathrm{k}}\right) g_{k}\left(x, u_{k}\right) \zeta\right) d x \leq \int_{\bar{\Omega}} \zeta d|\omega|
$$

and

$$
\left\|u_{k}\right\|_{L^{1}}+\left\|\rho g_{k}\left(., u_{k}\right)\right\|_{L_{\rho}^{1}} \leq C_{1}\|\omega\|_{\mathfrak{M}_{\rho}} .
$$

Furthermore, by estimates of Proposition 2.5 and since $\left|u_{k}\right| \leq \overline{\mathbb{G}}[|\omega|]$, there holds,

$$
\left\|u_{k}\right\|_{M_{\rho}^{(N+1) / N}}+\left\|\nabla u_{k}\right\|_{M_{\rho}^{(N+1) / N}} \leq C\|\omega\|_{\mathfrak{M}_{\rho}} .
$$

Since the right-hand side of (3.65) is bounded independently of $k$ fixed, there exist a subsequence $\left\{u_{k_{j}}\right\}$ and a function $u \in W_{l o c}^{1, q}(\Omega)$, for any $1 \leq q<(N+1) / N$, such that $u_{k_{j}} \rightarrow u$ a.e. in $\Omega$ and thus $g_{k_{j}} \circ u_{k_{j}} \rightarrow g \circ u$ a.e. - and weakly in $W_{l o c}^{1, q}(\Omega)$ when $k_{j} \rightarrow \infty$. Let $R>0$ and $E \subset \Omega$ be a Borel subset, then

$$
\begin{aligned}
\int_{E}\left|g_{k_{j}} \circ u_{k_{j}}\right| \rho d x & \leq \int_{E \cap\left\{\left|u_{k_{j}}\right| \leq R\right\}} \tilde{g}\left(\left|u_{k_{j}}\right|\right) \rho h d x+\int_{E \cap\left\{\left|u_{k_{j}}\right|>R\right\}} \tilde{g}\left(\left|u_{k_{j}}\right|\right) \rho h d x \\
& \leq \tilde{g}(R) \int_{E} \rho h d x-\int_{R}^{\infty} \tilde{g}(s) d \theta_{u_{k_{j}}}(s),
\end{aligned}
$$

where, we recall it,

$$
\theta_{u_{k_{j}}}(s):=\int_{\lambda_{u_{k_{j}}}(s)} d(\rho h) .
$$


Since $\left|u_{k_{j}}\right| \leq \overline{\mathbb{G}}[|\omega|], \theta_{u_{k_{j}}}(s) \leq \theta_{\overline{\mathbb{G}}[|\omega|]}(s)$. By assumption,

$$
\theta_{\overline{\mathbb{G}}\left[\left|\omega_{n}\right|\right]}(s) \leq C \Psi(s) \quad \forall s>0,
$$

with

$$
C=C_{\rho h}^{\Psi}(\overline{\mathbb{G}}[|\omega|]) .
$$

Furthermore, by a standard integration by parts in Stieltjes integrals and for a.e. $R$,

$$
\begin{aligned}
-\int_{R}^{\infty} \tilde{g}(s) d \theta_{u_{k_{j}}}(s) & \left.=\tilde{g}(R) \theta_{u_{k_{j}}}(R)+\int_{R}^{\infty} \theta_{u_{k_{j}}}(s) d \tilde{g}(s)\right) \\
& \leq \tilde{g}(R) \theta_{u_{k_{j}}}(R)+C \int_{R}^{\infty} \Psi(s) d \tilde{g}(s) \\
& \leq \tilde{g}(R) \theta_{u_{k_{j}}}(R)-C \tilde{g}(R) \Psi(R)-C \int_{R}^{\infty} \tilde{g}(s) d \Psi(s) \\
& \leq-C \int_{R}^{\infty} \tilde{g}(s) d \Psi(s)
\end{aligned}
$$

Since condition (3.58) holds, it follows

$$
\lim _{R \rightarrow \infty} \int_{R}^{\infty} \tilde{g}(s) d \Psi(s)=0
$$

Given $\epsilon>0$, we first choose $R>0$ such that

$$
-C \int_{R}^{\infty} \tilde{g}(s) d \Psi(s) \leq \epsilon / 2
$$

Then we put $\delta=\epsilon /(2(1+\tilde{g}(R))$ and derive

$$
\int_{E} \rho d x \leq \delta \Longrightarrow \int_{E}\left|g_{k_{j}}\left(u_{k_{j}}\right)\right| \rho h d x \leq \epsilon .
$$

Therefore $\left\{g_{k_{j}} \circ u_{k_{j}}\right\}$ is uniformly integrable in $L_{\rho}^{1}(\Omega)$. It follows by Vitali's convergence theorem

$$
\lim _{k \rightarrow \infty} g_{k_{j}} \circ u_{k_{j}}=g \circ u \quad \text { in } L_{\rho}^{1}(\Omega) .
$$

Let $\zeta \in C_{c}^{1, L}(\bar{\Omega})$. If we let $k_{j} \rightarrow \infty$ in the equality

$$
\int_{\Omega}\left(-u_{k_{j}} L^{*} \zeta+g_{k_{j}} \circ u_{k_{j}} \zeta\right) d x=\int_{\bar{\Omega}} \zeta d \omega
$$

we derive

$$
\int_{\Omega}\left(-u L^{*} \zeta+g \circ u \zeta\right) d x=\int_{\bar{\Omega}} \zeta d \omega .
$$

Uniqueness follows classicaly if $g(x,$.$) is nonndecreasing.$

The following extension of the previous result is an adaptation of [13, Th. 3.20] 
Theorem 3.4 Assume $g$ belongs to the class $G_{h, \Psi}$ and satisfies the following $\Delta_{2}$-condition

$$
\left|g\left(x, r+r^{\prime}\right)\right| \leq \theta\left(|g(x, r)|+\left|g\left(x, r^{\prime}\right)\right|\right)+\ell(x) \quad \forall x \in \Omega, \forall\left(r, r^{\prime}\right) \in \mathbb{R} \times \mathbb{R},
$$

for some nonnegative $\ell \in L_{\rho}^{1}(\Omega)$. Suppose also that $r \mapsto g(x, r)$ is nondeacreasing. If $\omega \in \mathfrak{M}_{\rho}(\bar{\Omega})$ has Lebesgue decomposition $\omega=\omega_{r}+\omega_{s}$ with regular part with respect to the Lebesgues measures $\omega_{r}$ and singular part $\omega_{s}$, and if $\omega_{s}$ belongs to $B_{h}^{\Psi}(\bar{\Omega})$, then there exists a unique solution $u$ to (3.60).

Proof. If $g$ satisfies (3.72), $g_{k}$ defined by (3.61) shares the same property with the same $\ell$. Therefore, by [13, Th 3.12], there exists a solution $u_{k}$ to (3.62). Actually, in this result it is only assume that $\ell$ in (3.72) is a constant, but the proof is valid if it is a nonnegative function in $L_{\rho}^{1}(\Omega)$. Let $v_{k}$ and $v_{k}^{\prime}$ be weak solutions in $\bar{\Omega}$ of $-L v_{k}+g_{k} \circ v_{k}=\omega_{r}^{+}$and $-L v_{k}^{\prime}-g_{k} \circ\left(-v_{k}^{\prime}\right)=\omega_{r}^{-}$ respectively. Set $w_{k}=v_{k}+\overline{\mathbb{G}}\left(\omega_{s}^{+}\right)$and $w_{k}^{\prime}=v_{k}^{\prime}+\overline{\mathbb{G}}\left(\omega_{s}^{-}\right)$. Then $-L w_{k}+g_{k} \circ w_{k} \geq \omega^{+}$and $-L w_{k}^{\prime}-g_{k} \circ\left(-w_{k}^{\prime}\right) \geq \omega^{-}$in $\bar{\Omega}$. By monotonicity $-w_{k}^{\prime} \leq u_{k} \leq w_{k}$, thus $g_{k}\left(-w_{k}^{\prime}\right) \leq g_{k}\left(u_{k}\right) \leq$ $g_{k}\left(w_{k}\right)$. The estimates (3.64) and (3.65) are satisfied, therefore there exist a function $u \in L^{1}(\Omega)$ and a subsequence $u_{k_{j}}$ which converges to $u$ a.e. in $\Omega$. Furthermore

$$
\begin{aligned}
g_{k}\left(x, u_{k}\right) & \leq \theta\left(g_{k}\left(x, v_{k}\right)+g_{k}\left(x, \overline{\mathbb{G}}\left(\omega_{s}^{+}\right)\right)+\ell\right. \\
& \leq \theta\left(g_{k}\left(x, v_{k}\right)+g\left(x, \overline{\mathbb{G}}\left(\omega_{s}^{+}\right)\right)+\ell\right.
\end{aligned}
$$

Since the sequence $\left\{\left|g_{k}\right|\right\}$ increases, $\left\{v_{k}\right\}$ and $\left\{v_{k}^{\prime}\right\}$ decrease. Therefore $v_{k} \downarrow v$ and $v_{k}^{\prime} \downarrow v^{\prime}$ which satisfy $-L v+g \circ v=\omega_{r}^{+}$and $-L v^{\prime}-g_{k} \circ\left(-v^{\prime}\right)=\omega_{r}^{-}$respectively in $\bar{\Omega}$. Therefore $g_{k} \circ v_{k} \rightarrow g \circ v$ and $g_{k} \circ v_{k}^{\prime} \rightarrow-g \circ\left(-v^{\prime}\right)$ in $L_{\rho}^{1}(\Omega)$ respectively. Since

$$
g_{k} \circ \overline{\mathbb{G}}\left(\omega_{s}^{+}\right) \leq g \circ \overline{\mathbb{G}}\left(\omega_{s}^{+}\right)
$$

and $\omega_{s} \in B_{h}^{\Psi}(\bar{\Omega}), g \circ \overline{\mathbb{G}}\left(\omega_{s}^{+}\right.$by Lemma 3.2, the right-hand side term of inequality (3.73) is uniformly integrable in $L_{\rho}^{1}(\Omega)$. Similarly

$$
g_{k}\left(x, u_{k}\right) \geq \theta\left(g_{k}\left(x,-v_{k}^{\prime}\right)+g\left(x,-\overline{\mathbb{G}}\left(\omega_{s}^{-}\right)\right)-\ell\right.
$$

and the right-hand side of (3.74) is also uniformly integrable in $L_{\rho}^{1}(\Omega)$. We conclude as in Theorem 3.3 .

\section{Stability}

Lemma 4.1 Let $\left\{\omega_{n}\right\} \subset B_{h}^{\Psi}(\bar{\Omega})$ be a sequence of measures such that $C_{\rho}^{\Psi}\left(\overline{\mathbb{G}}\left[\left|\omega_{n}\right|\right]\right)$ is bounded independently of $n$. Then $\left\{\omega_{n}\right\}$ remains bounded in $\mathfrak{M}_{\rho}(\bar{\Omega})$. If $\omega_{n} \rightarrow \omega$ weakly in $\mathfrak{M}_{\rho}(\bar{\Omega})$, then $\omega \in B_{h}^{\Psi}(\bar{\Omega})$.

Proof. Since $C_{\rho}^{\Psi}\left(\overline{\mathbb{G}}\left[\left|\omega_{n}\right|\right]\right)$ is uniformly bounded, the sequence $\left.\left\{g \circ \overline{\mathbb{G}}\left[\left|\omega_{n}\right|\right]\right)\right\}$ is bounded in $L_{\rho}^{1}(\Omega)$ by Lemma [3.2. Since $\omega_{n} \rightarrow \omega$ weakly in $\mathfrak{M}_{\rho}(\bar{\Omega}), \overline{\mathbb{G}}\left[\omega_{n}\right] \rightarrow \overline{\mathbb{G}}[\omega]$ in $L_{\rho}^{1}(\Omega)$ and, up to a subsequence, a.e. in $\Omega$. Therefore, and up to sets of zero Lebesgue measure, 


$$
\lambda_{\overline{\mathbb{G}}[\omega]}(t) \subset \bigcap_{n \geq 0}\left(\bigcup_{p \geq n} \lambda_{\overline{\mathbb{G}}\left[\omega_{p}\right]}(t)\right) \subset \bigcap_{n \geq 0}\left(\bigcup_{p \geq n} \bar{\lambda}_{\overline{\mathbb{G}}\left[\omega_{p}\right]}(t)\right) \subset \bar{\lambda}_{\overline{\mathbb{G}}[\omega]}(t) .
$$

Therefore

$$
\limsup _{n \rightarrow \infty} \theta_{\lambda_{\bar{G}\left[\omega_{n}\right]}(t)} \leq \theta_{\bar{\lambda}_{\bar{G}[\omega]}(t)} .
$$

Conversely, for any $x \in \lambda_{\overline{\mathbb{G}}[\omega]}(t)$, i.e. such that $\overline{\mathbb{G}}[\omega](x)>t$, there exists $n_{x}$ such that $x \in \lambda_{\overline{\mathbb{G}}\left[\omega_{n}\right]}(t)$ if $n \geq n_{x}$. This implies

$$
\lim _{n \rightarrow \infty} \chi_{\lambda_{\overline{\mathbb{G}}[\omega n]}(t)} \chi_{\lambda_{\overline{\mathbb{G}}[\omega]}(t)}=\chi_{\lambda_{\overline{\mathbb{G}}[\omega]}(t)},
$$

and

$$
\liminf _{n \rightarrow \infty} \theta_{\lambda_{\bar{G}\left[\omega_{n}\right]}(t)} \geq \theta_{\lambda_{\bar{G}[\omega]}(t)} .
$$

Since $\theta_{\lambda_{\overline{\mathbb{G}}\left[\omega_{n}\right]}(t)} \leq C_{\rho}^{\Psi}\left(\overline{\mathbb{G}}\left[\left|\omega_{n}\right|\right]\right) \Psi(t)$ and the $C_{\rho}^{\Psi}\left(\overline{\mathbb{G}}\left[\left|\omega_{n}\right|\right]\right)$ are bounded, it follows that $\omega$ belongs to $B_{h}^{\Psi}(\bar{\Omega})$.

Theorem 4.2 Assume $g$ belongs to the class $G_{h, \Psi}$ and $r \mapsto g(x, r)$ is nondecreasing for a.e. $x \in$ $\Omega$. Let $\left\{\omega_{n}\right\} \subset B_{h}^{\Psi}(\bar{\Omega})$ be a sequence of measures such that $C_{\rho}^{\Psi}\left(\mathbb{G}\left[\left|\omega_{n}\right|\right]\right)$ is bounded independently of $n$ which converges to $\omega$ weakly with respect to $C_{1}(\bar{\Omega})$. Then the solution $u_{n}$ of

$$
-L u_{n}+g \circ u_{n}=\omega_{n} \quad \text { in } \bar{\Omega}
$$

converges to the solution $u$ of

$$
-L u+g \circ u=\omega \quad \text { in } \bar{\Omega}
$$

Proof. Since $u_{n}$ satisfies the Brezis estimates (3.64) and (3.65), there exists a subsequence $\left\{u_{n_{j}}\right\}$ and $u \in L^{1}(\Omega)$ such that $u_{n_{j}} \rightarrow u$ a.e. in $\Omega$ and in $L^{1}(\Omega)$. As in the proof of Theorem 3.3 , the problem is to prove the convergence of the $g \circ u_{n_{j}}$ in $L_{\rho}^{1}(\Omega)$. But this is a clearly obtained by the uniform integrability, as in the proof of Theorem 3.3-Step 2, using the fact that, in (3.67), the $\theta_{u_{n_{j}}}$ are bounded by $\sup _{n} C_{\rho h}^{\Psi}\left(\overline{\mathbb{G}}\left[\omega_{n}\right]\right) \Psi$.

Theorem 4.3 Assume $g$ belongs to the class $G_{h, \Psi}$, satisfies the $\Delta_{2}$-condition (3.72) and $r \mapsto$ $g(x, r)$ is nondeacreasing. Let $\left\{\omega_{n}\right\} \subset \mathfrak{M}_{\rho}(\bar{\Omega})$ has Lebesgue decomposition $\omega_{n}=\omega_{n r}+\omega_{n s}$ if $\left\{\omega_{n s}\right\} \subset B_{h}^{\Psi}(\bar{\Omega})$ are such that the $C_{\rho h}^{\Psi}\left(\overline{\mathbb{G}}\left[\omega_{n s}\right]\right)$ are uniformly bounded, then the solutions $u_{n}$ of (4.78) converges in $L^{1}(\Omega)$ to the solution $u$ of (4.79).

Proof. The argument follows the one of Theorem 3.4. Let $v_{n}$ and $v_{n}^{\prime}$ be weak solutions in $\bar{\Omega}$ of $-L v_{n}+g \circ v_{n}=\omega_{n r}^{+}$and $-L v_{n}^{\prime}-g \circ\left(-v_{n}^{\prime}\right)=\omega_{n r}^{-}$respectively. Set $w_{n}=v_{n}+\overline{\mathbb{G}}\left(\omega_{n s}^{+}\right)$and $w_{k}^{\prime}=v_{k}^{\prime}+\overline{\mathbb{G}}\left(\omega_{n s}^{-}\right)$. Then $-L w_{n}+g \circ w_{n} \geq \omega_{n}^{+}$and $-L w_{n}^{\prime}-g \circ\left(-w_{n}^{\prime}\right) \geq \omega_{n}^{-}$. By monotonicity $-w_{n}^{\prime} \leq u_{n} \leq w_{n}$, thus $g\left(-w_{n}^{\prime}\right) \leq g\left(u_{n}\right) \leq g\left(w_{n}\right)$. The estimates (3.64) and (3.65) are satisfied therefore there exist a function $u \in L^{1}(\Omega)$ and a subsequence $u_{n_{j}}$ which converges to $u$ a.e. in $\Omega$ and in $L^{1}(\Omega)$. Furthermore

$$
\begin{aligned}
g\left(x, u_{n}\right) & \leq \theta\left(g\left(x, v_{n}\right)+g\left(x, \overline{\mathbb{G}}\left(\omega_{n s}^{+}\right)\right)+\ell\right. \\
& \leq \theta\left(g\left(x, v_{n}\right)+g\left(x, \overline{\mathbb{G}}\left(\omega_{n s}^{+}\right)\right)+\ell .\right.
\end{aligned}
$$


Classicaly $v_{n} \rightarrow v v_{n}^{\prime} \rightarrow v^{\prime}$ in $L^{1}(\Omega)$ which satisfy $-L v+g \circ v=\omega_{r}^{+}$and $-L v^{\prime}-g_{k} \circ\left(-v^{\prime}\right)=\omega_{r}^{-}$ respectively. Therefore $g \circ v_{n} \rightarrow g \circ v$ and $g \circ v^{\prime} \rightarrow-g \circ\left(-v^{\prime}\right)$ in $L_{\rho}^{1}(\Omega)$ respectively. Since $C_{\rho h}^{\Psi}\left(\overline{\mathbb{G}}\left[\omega_{n s}\right]\right)$ is uniformly bounded the $g \circ \overline{\mathbb{G}}\left[\omega_{n s}\right]$ are uniformly integrable in $L_{\rho}^{1}(\Omega)$ by Lemma 3.2 . Therefore the $\left(g \circ u_{n}\right)^{+}$are uniformly integrable in $L_{\rho}^{1}(\Omega)$. Similarly

$$
g\left(x, u_{n}\right) \geq \theta\left(g\left(x,-v_{k}^{\prime}\right)+g\left(x,-\overline{\mathbb{G}}\left(\omega_{s}^{-}\right)\right)-\ell\right.
$$

and the $\left(g \circ u_{n}\right)^{-}$are also uniformly integrable in $L_{\rho}^{1}(\Omega)$. The conclusion follows in the same way as in Theorem 3.4 .

\section{References}

[1] Adams D. R., Hedberg L. I.: Function spaces and potential theory Grundlehren Math. Wissen. 314, Springer (1996).

[2] Ancona A.:Principe de Harnak à la frontière et théorème de Fatou pour un opérateur elliptique dans un domaine Lipschitzien, Ann. Inst. Fourier (Grenoble) 28, 169-213 (1978).

[3] Bénilan Ph., Brezis H.: Nonlinear problems related to the Thomas-Fermi equation, J. Evolution Eq. 3, 673-770 (2003).

[4] Bénilan Ph., Brezis H., Crandall M.: A semilinear elliptic equation in $L^{1}\left(\mathbb{R}^{N}\right)$, Ann. Sc. Norm. Sup. Pisa Cl. Sci. 5 Vol. 2, 523555 (1975).

[5] Bidaut-Véron M.F., Vivier L. : An elliptic semilinear equation with source term involving boundary measures: The subcritical case, Rev. Mat. Iberoamericana 16, 477513 (2000).

[6] Brezis H.: Some variational problems of the Thomas-Fermi type. Variational inequalities and complementarity problems, Proc. Internat. School, Erice, 1978, pp. 53-73, Wiley, Chichester (1980).

[7] Gmira A., Véron L.: Boundary singularities of solutions of nonlinear elliptic equations, Duke J. Math. 64, 271-324 (1991).

[8] Marcus M.: Stability relative to weak convergence for a family of semilinear elliptic equations with measure data, preprint (2012).

[9] Marcus M., Véron L.: A characterization of Besov spaces with negative exponents, Around the Research of Vladimir Maz'ya I. Function Spaces, Springer Verlag International Mathematical Series, Vol. 11, 273-284 (2010).

[10] Pinchover Y., On positive solutions of second-order elliptic equations, stability results, and classification, Duke Math. J. 57, 955-980 (1988).

[11] Triebel H., Interpolation Theory, Function Spaces, Differential Operators, North Holland Publ. Co. (1978). 
[12] Véron L.: Weak and strong singularities of nonlinear elliptic equations, Proc. Symposia in Pure Math. 45 Part 2, 477-495 (1986).

[13] Véron L.: Elliptic equations involving measures in Stationary partial differential equations Vol. I, 593-712. Handb. Differ. Equ., North-Holland, Amsterdam (2004).

[14] Véron L.: Singularities of Solutions of Second Order Quasilinear Equations, Pitman Research Notes in Math. Vol. 353, Longman, Harlow (1996). 